\title{
ERRATUM
}

\section{Linear interaction of two-dimensional free-stream disturbances with an oblique shock wave - ERRATUM}

\author{
Zhangfeng Huang and Huilin Wang \\ doi:10.1017/jfm.2019.438, Published online by Cambridge University Press,
} 1 July 2019

The above-named article was originally published with Zhangfeng Huang's name given as 'Zhengfeng Huang'.

Cambridge University Press apologises for this error which has since been rectified.

\section{REFERENCE}

HUANG, Z. \& WANG, H. 2019 Linear interaction of two-dimensional free-stream disturbances with an oblique shock wave. J. Fluid Mech. 873, 1179-1205. 